\title{
A Circularly Polarized Wireless Power Transfer System for Internet-of-Things (IoT) Applications
}

\author{
Wei Lin and Richard W. Ziolkowski \\ Global Big Data Technologies Centre, \\ School of Electrical and Data Engineering, \\ University of Technology Sydney, \\ Ultimo 2007, Sydney, Australia, \\ Email: Wei.Lin@uts.edu.au, Richard.Ziolkowski@uts.edu.au
}

\begin{abstract}
A circularly polarized (CP) wireless power transfer (WPT) system is demonstrated. It consists of a high directivity, omnidirectional CP (OCP) antenna array and multiple electrically small Huygens CP (HCP) rectennas. The OCP array emits highly directional $C P$ fields over the entire azimuthal plane. It acts as the wireless power source for charging or powering up surrounding IoT sensors. The OCP array is realized by cascading multiple vertical bars and helical loops to form a set of properly phased, collinear array of electric and magnetic dipoles. Wirelessly powered (WP) sensors are facilitated by combining sensors with HCP rectennas, the latter being a seamless integration of an electrically small HCP antenna with highly efficient rectifier circuits. Both systems were tested and achieved their predicted performance characteristics. These WP sensor systems are ideal candidates for wireless internet-of-things (IoT) applications, for example, in the food and agriculture industries, which are amongst the largest enterprises in Australia.
\end{abstract}

Keywords-antenna array; circular polarization; electrically small antennas; omnidirectional; rectenna; wireless power transfer.

\section{INTRODUCTION}

The food and agriculture industries are some of the largest enterprises in Australia. Agriculture digitalization and smart farms are becoming more and more attractive along with the rapid development of wireless internet-of-thing (IoT) systems facilitated by the upcoming $5 \mathrm{G}$ technologies. IoT sensor-based smart farms will help increase agricultural productivity and quality. The measured data from IoT sensors such as soil condition, PH level, moisture level, temperature, humidity, livestock body conditions and locations, etc., can be analyzed for optimal decision making. Given the anticipated large numbers of IoT sensors associated with this application, wirelessly powered (batteryless) IoT sensors are desired for the future smart agriculture ecosystem.

Fig. 1 illustrates a circularly polarized (CP) wireless power transfer (WPT) system for an IoT sensor-based smart agriculture scheme. CP systems are preferred in WPT applications as they have been demonstrated to avoid polarization mismatch issues in many wireless applications, e.g., device-to-device (D2D) communications [1], RFID systems [2], space-to-ground data links [3], urban microcell base stations [4], and millimeter-wave systems [5]. This example system consists of two major subsystems. One is a high directivity, omnidirectional CP (OCP) antenna array that emits $\mathrm{CP}$ wireless power in $360^{\circ}$ directions to charge or power up the surrounding IoT sensors. Another is a network of electrically small Huygens CP (HCP) rectennas integrated with the IoT sensors; they convert the wireless power to DC energy. This paper will discuss the design, operating principles, and measured results of both subsystems.

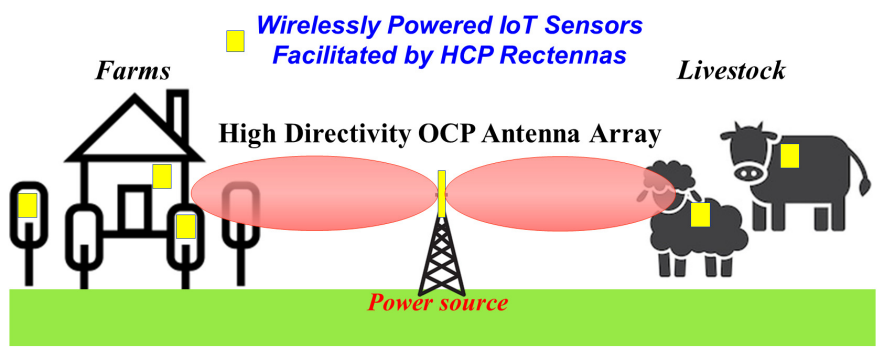

Figure 1. Illustrated CP wireless power transfer system for IoT applications in the food and agriculture industries.

\section{Design And Realization of the High DiRectivity, OMNIDIRECTIONAL CP (OCP) ANTENNA ARRAY}

\section{A. Array Design and Operating Principles}

The configuration of the high directivity, OCP antenna array is shown in Fig. 2. It consists of six stages of cascaded vertical metal bars and helical loops. It is a compact system that is realized with 3D printing technology. A microstrip balun is adopted to excite symmetrical radiators in the middle. The indicated current distributions show that a set of collinear electric and magnetic dipole radiators have been formed. The natural phase difference between the electric and magnetic dipoles is $90^{\circ}$. The detailed descriptions of the OCP array are given in reference [6].

\section{B. Measured performance}

The measured $\left|\mathrm{S}_{11}\right|$, realized gain, and $A R$ values of the OCP array are shown in Fig. 2. The operational bandwidth covers $110 \mathrm{MHz}$ from 2.37 to $2.48 \mathrm{GHz}$ with the peak realized gain of $7.12 \mathrm{dBic}$. The measured radiation patterns are given in Fig. 3. The measured results agree very well with their simulated values. High directivity OCP radiation was realized. Due to the simplicity of the structure, it is straightforward to redesign to other frequencies, e.g., $915 \mathrm{MHz}$ in the ISM band. 


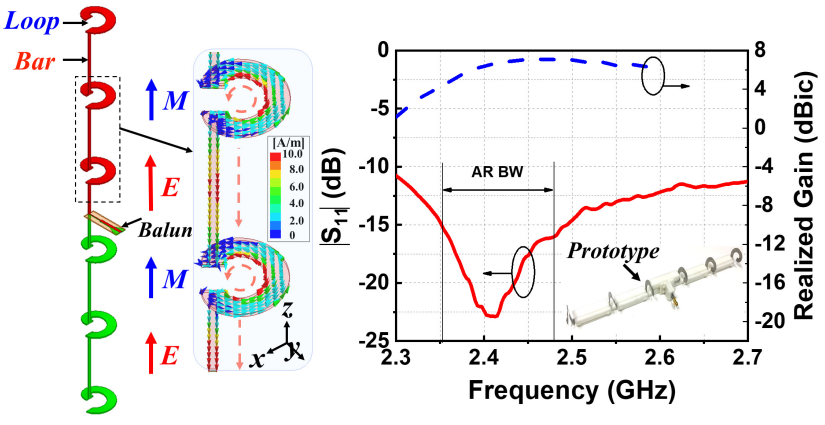

Figure 2. High directivity OCP array configuration and the measured $\left|S_{11}\right|$, realized gain, and axial ratio $(\mathrm{AR})$ values.
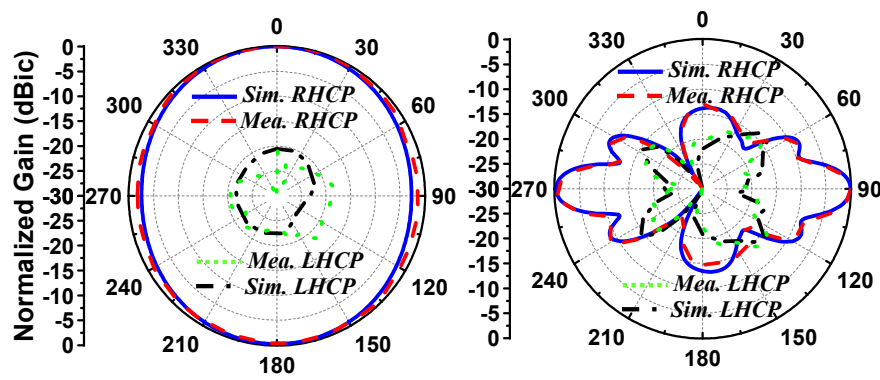

Figure 3. Measured realized gain patterns in both the horizontal and vertical planes at the center frequency illustrate the realized high directivity, omnidirectional beams.

\section{Design And Realization OF THE EleCtrically SMALl HUYGENS CP (HCP) RECTENNA}

\section{A. HCP Rectenna System Design and Operating Principles}

A rectenna, i.e., an antenna integrated with a rectifier circuit, is the key component for far-field WPT applications [7]-[9]. An electrically small Huygens CP rectenna was developed, as shown in Fig. 4, for powering IoT sensors. It is the seamless integration of an $\mathrm{HCP}$ receiving antenna with a highly efficient rectifier circuit. The HCP antenna consists of two pairs of metamaterial-inspired near field resonant parasitic (NFRP) elements, i.e., crossed Egyptian axe dipoles (EADs) and crossed capacitively loaded loops (CLLs), driven by a crossed dipole antenna. A delayed loop feedline is adopted to achieve an inductive impedance that conjugately matches the HCP antenna directly to the capacitive rectifier circuit. The rectifier is highly compact and vertically connected to the HCP antenna. The detailed parameters of this HCP rectenna design are found in [10].

\section{B. Simulated and Measured Performance}

The HCP receiving antenna has a cardioid-shaped unidirectional CP pattern with wide beamwidth. Thus, the developed rectenna has a broad range of wireless power capture angles. The simulated electromagnetic field power flow into the HCP rectenna shown in Fig. 5 demonstrates that it has a large capture region, more than two times larger than a dipole rectenna with the same length. The measured peak AC to DC conversion efficiency agrees reasonably well with its simulated value. The delayed loop feedline feature eliminates a lossy inductor allowing the rectenna to obtain the maximum achievable AC to DC conversion efficiency [7].

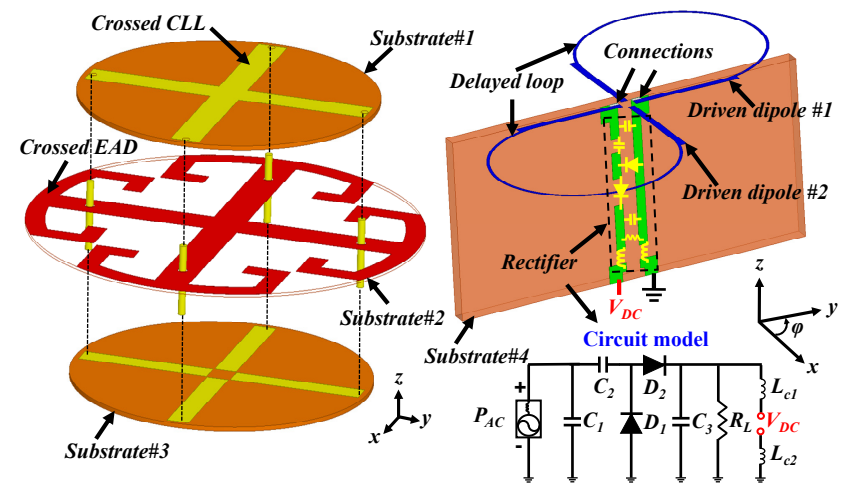

Figure 4. Configuration of the electrically small Huygens CP rectenna with its delayed-loop crossed-dipole driven element.
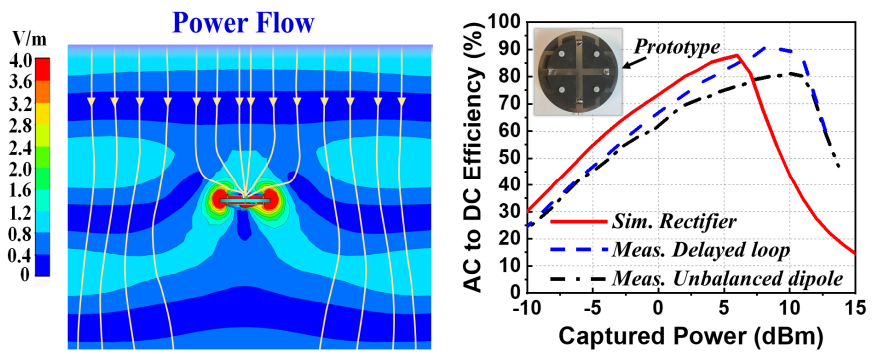

Figure 5. Simulated power flow capture region of the electrically small HCP antenna and its measured AC to DC conversion efficiency.

\section{REFERENCES}

[1] M. N. Tehrani, M. Uysal and H. Yanikomeroglu, "Device-to-Device communication in $5 \mathrm{G}$ cellular networks: Challenges, solutions, and future directions," IEEE Commun. Mag., vol. 52, no. 5, Feb. 2014, pp. 86-92.

[2] X. Liu, Y. Liu, and M. M. Tentzeris, "A novel circularly polarized antenna with coin-shaped patches and a ring-shaped strip for worldwide UHF RFID applications," IEEE Antennas Wireless Propag. Lett., vol. 14, pp. 707-710, Sep. 2015.

[3] G. Minatti, S. Maci, P. De Vita, A. Freni, and M. Sabbadini, "A circularly-polarized isoflux antenna based on anisotropic metasurface," IEEE Trans. Antennas Propag., vol. 60, no. 11, pp. 4998-5009, Nov. 2012.

[4] Y. Qin, X. Mo, R. Li, T. Wong and Y. Cui, "A broadband wide-beam circularly polarized antenna array for urban macrocell base stations", IEEE Trans. Antennas Propag., early access, Dec. 2019.

[5] Y. Li, Z. N. Chen, X. M. Qing, Z. J. Zhang, J. F. Xu, and Z. H. Feng, "Axial ratio bandwidth enhancement of $60-\mathrm{GHz}$ substrate integrated waveguide-fed circularly polarized LTCC antenna array," IEEE Trans. Antennas Propag., vol. 60, no. 10, pp. 4619-4626, Oct. 2012.

[6] W. Lin and R. W. Ziolkowski, "Compact, high directivity, omnidirectional circularly polarized antenna array," IEEE Trans. Antennas Propag., vol. 67, no. 7, pp. 4537-4547, July 2019.

[7] W. Lin, R. W. Ziolkowski and J. Q. Huang, "Electrically small, highly efficient, Huygens dipole rectennas for wirelessly powering Internet-ofThings (IoT) devices," IEEE Trans. Antennas Propag., vol. 67, no. 6, pp. 3670-3679, June 2019.

[8] W. Lin and R. W. Ziolkowski, "Electrically small Huygens antennabased fully-integrated wireless power transfer and communication system,” IEEE Access, vol. 7, pp. 39762-39769, Mar. 2019.

[9] W. Lin and R. W. Ziolkowski, "Wirelessly powered temperature and light detecting sensors based on electrically small Huygens antennas," Sensors, vol. 19, no. 9, Apr. 2019.

[10] W. Lin and R. W. Ziolkowski, "Electrically small Huygens CP rectenna with a driven loop element maximizes its wireless power transfer efficiency," IEEE Trans. Antennas Propag., early access. 2019. 